Document downloaded from:

http://hdl.handle.net/10251/120144

This paper must be cited as:

Sanchez-Caballero, S.; Sellés Cantó, MÁ.; Ferrándiz Bou, S.; Peydro, MA.; Oliver, B. (2018). Failure analysis of a plastic modular belt in-service. Engineering Failure Analysis. 93:13-25. https://doi.org/10.1016/j.engfailanal.2018.06.019

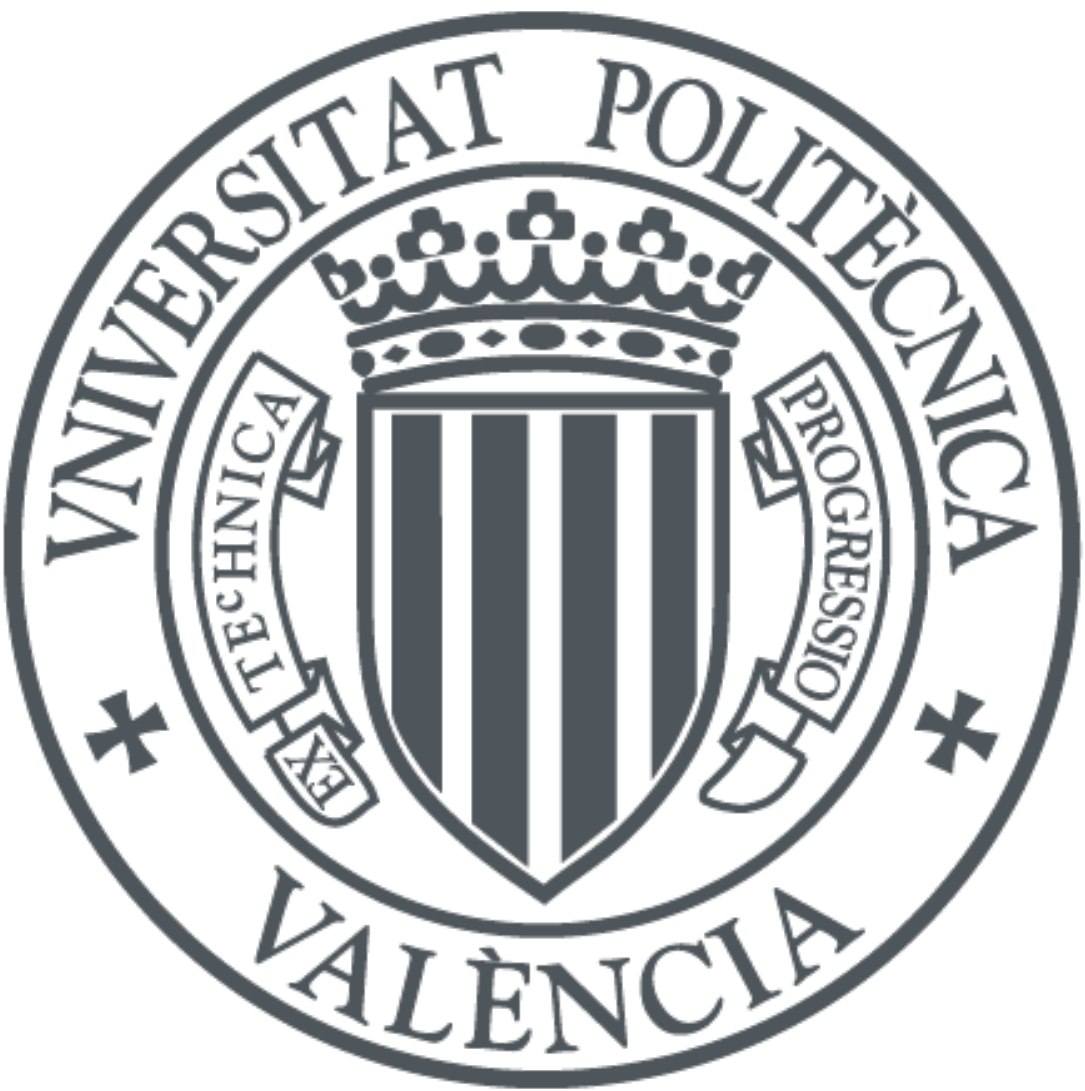

The final publication is available at

https://doi.org/10.1016/j.engfailanal.2018.06.019

Copyright Elsevier

Additional Information 


\title{
Failure analysis of a plastic modular belt in-service
}

S. Sanchez-Caballero*, M.A. Selles, S. Ferrandiz, M.A. Peydro, B.A. Oliver

Department of Mechanical Engineering and Materials, Universitat Politecnica de Valencia (Campus de Alcoy). Plaza Ferrandiz y Carbonell, s/n 03801 Alcoy, Spain

\begin{abstract}
In this study, an analysis of the possible causes of the failure in-service of a section of a plastic modular belt was conducted. The study begins with a reproduction of the service conditions in a traction gear. An analysis of the fracture surfaces revealed the existence of defects in the interior of the parts. With the aim of determining the origin of the imperfections and their influence on the failure, an exhaustive mechanical and rheological characterization of the material was carried out. The development of an FE Analysis established that the reduction of the tensile strength of the part due to internal defects was around $70 \%$. Tests also showed that the most stressed area was the area where the most defects appeared. A simulation of the injection process showed that the defects are caused by the geometry of the part, leading to the conclusion that its failure was caused by bad dimensioning of thicknesses.

Keywords: Plastic modular belt, belt link, Tensile test, Rheology, Injection simulation, Defects, Shrink cavity, Air traps
\end{abstract}

\section{Introduction}

Plastic modular belts (Figure 1) are widely used in industry as a means of transporting material during production processes. Their niche in the market is located between metal and belt conveyors. The main advantages of this type

5 of system are its capacity to deal with curved trajectories, its lightness, its

*Corresponding author
Email address: sasanca@dimm.upv.es (S. Sanchez-Caballero)

Preprint submitted to Engineering Failure Analysis

June 14, 2018 
mechanical and chemical strength and that it is ease to repair. Because of their construction, they are especially suited to humid or saline conditions, and food and chemical industries.
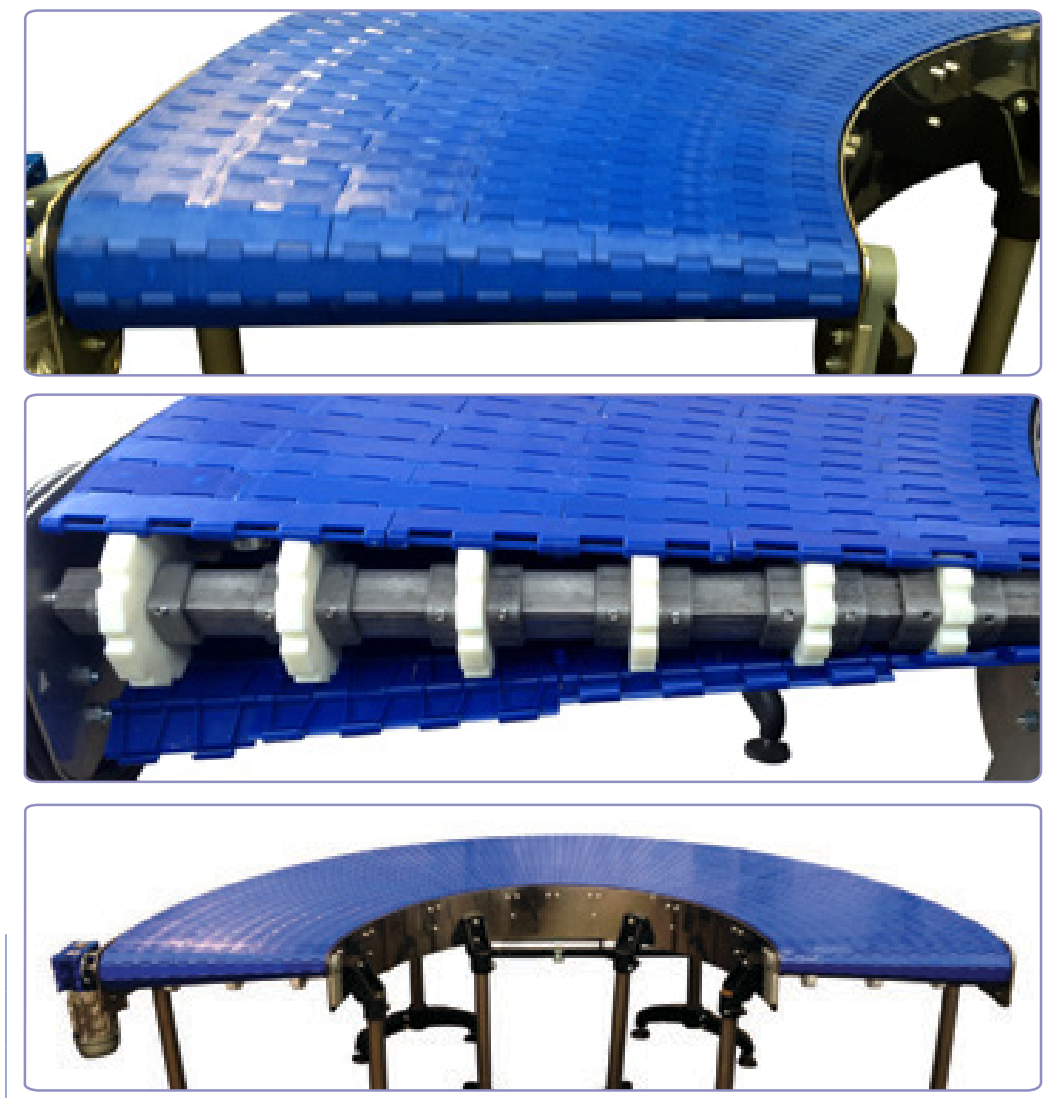

Figure 1: Plastic modular belt.

These types of belts are made up of a number of links that are connected by a series of pins to form the width of the belt. This width may vary from a few centimeters to several meters, and thus, in order to achieve the desired width a number of links must be used adjacently. These links act simultaneously as handling and traction elements [1].

This study shows a real case of a fracture in-service in which the fracture 15 occurs in one of the thickest parts of the belt while carrying a load considerably 
lower than that recommended by the manufacturer. The belt links are made of an acetal copolymer, a material that has not been analyzed before in belt conveyors. There are several studies focused on other materials. Vaxman et al. [2], for example, worked on the void formation in thermoplastic composites; as well as Francis et al. [3], but they studied the failure analysis of a clamp hanger. Lewis 4] found that a premature fracture of a composite nylon radiator was caused by a bad quality control during injection moulding, rather than being a design fault. Other authors analyzed the failures of different parts made of PVC [5], HDPE [6] and aluminum alloys [7. All these studies follow a specific methodology that is going to be used in the present work as well.

The aim of the study is to determine the cause of the failure, if it was due to an inappropriate use of the belt, the material used in its manufacture, the manufacturing process used or the belt link design.

\section{Material and methods}

In order to establish the cause of failure, different tensile tests were carried out on the links of the modular belt. Following this, the tests results and the fracture surfaces were analyzed. Finally, a mechanical and rheological characterization of the material was performed with the aim of establishing the influence that the material properties and the processing techniques have on the tensile

\subsection{Belt link tensile test}

The first step in this study was to test the links' tensile strength. For that purpose, two clamps were designed in order set up the belt link in the same way as in on duty conditions. The tests were carried out at a speed of $1.2 \mathrm{~mm} / \mathrm{s}$. The

40 Data Acquisition System was composed of an MGCPLUS ${ }^{\circledR}$ (Hottinger Baldwin Messtechnik GmbH, Darmstadt,Germany) as DAS hardware, a 100 kN U3 load cell of the same brand, and a displacement transducer WS1.1-750-R1K-L10 (ASM GmbHb, Moosinning Germany). The time, force and movement data 
were acquired at a speed of $10 \mathrm{~Hz}$. Figure 2 shows one test in the tensile test machine.

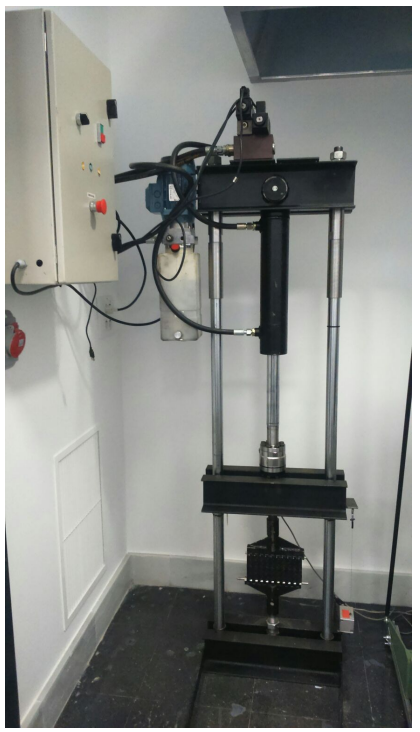

(a)

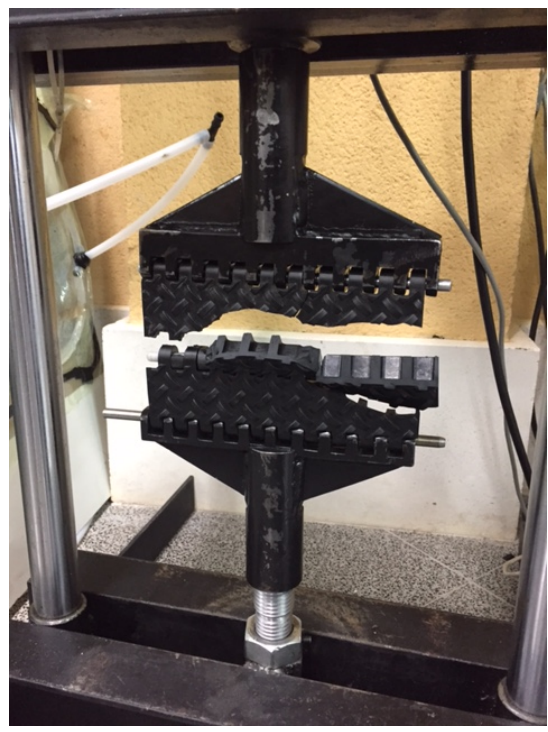

(b)

Figure 2: Tensile test of the modular belt link: a) setup overview b) detail

\subsection{Visual inspection}

After the belt link tests, pictures of the fracture surfaces were obtained using a OLYMPUS SZX7 stereo microscope with a OLYMPUS C-5060 Wide Zoom Camera, lighted with a OLYMPUS KL 1500 HL halogen light.

\subsection{Material tensile analysis}

In order to carry out a later Finite Element Analysis to quantify the loss of tensile strength of the part due to imperfections, a characterization of the material was done beforehand. The material used in the manufacture of the belt link was YUNCON ${ }^{\circledR}$ M90 (YunNan YunTianHua CO., YunNan ShuiFu, China),

${ }_{55}$ an Acetal (POM) Copolymer. Following the manufacturer's instructions, pellets were first dried in a dehumidifier MDEU1/10 (Industrial y Comercial Marse S.L., Barcelona Spain) at a temperature of $90{ }^{\circ} \mathrm{C}$ for four hours and the mold 
was tempered at a temperature of $70{ }^{\circ} \mathrm{C}$. Finally, a series of specimens was injected at $200 / 200 / 180 / 170{ }^{\circ} \mathrm{C}$ according to temperatures indicated by the manufacturer.

After the injection process, tensile tests were carried out on the specimens using a universal ELIB 30 tensile test machine supplied by S.A.E. Ibertest ${ }^{\circledR}$ (Madrid, Spain) following the guidelines of ISO 527, at a temperature of $25{ }^{\circ} \mathrm{C}$, with a relative humidity of $50 \%$ and with a $5 \mathrm{kN}$ load cell.

\subsection{The Finite Element Analysis}

Once the material was characterized, a static Finite Element Analysis was performed on the belt link in order to establish the theoretical value of the fracture load, in order to assess the influence of the imperfections associated to manufacturing process.

The three-dimensional model of the belt link, provided by the manufacturer, was generated using Solidworks ${ }^{\circledR}$. The CAD model was imported into Ansys ${ }^{\circledR}$ Workbench R18.2. and then meshed using tetrahedral elements (Tet10), yielding a total of 29,893 nodes and 16,291 elements. The material properties (Young's modulus, Poisson ratio and yield stress) used in the FEA were obtained 75 from the material tensile analysis.

Regarding to the load state, the lower holes were considered as fixed supports, while the remote displacement used in the FEA was considered as the maximum displacement obtained in the belt link test.

\subsection{Rheological characterization}

In order to assess the material behavior during the injection process, it is important to carry out a rheological analysis. The rheometry tests were carried out using a Thermo Haake Rheoflixer MT V, fulfilling ISO 11443. In order to simulate the temperatures recommended by the manufacturer, those used in the tests were 190 and $210{ }^{\circ} \mathrm{C}$. For each temperature three different nozzles were used, with shear rates ranging from 100 to $5.000 \mathrm{~s}^{-1}$. 


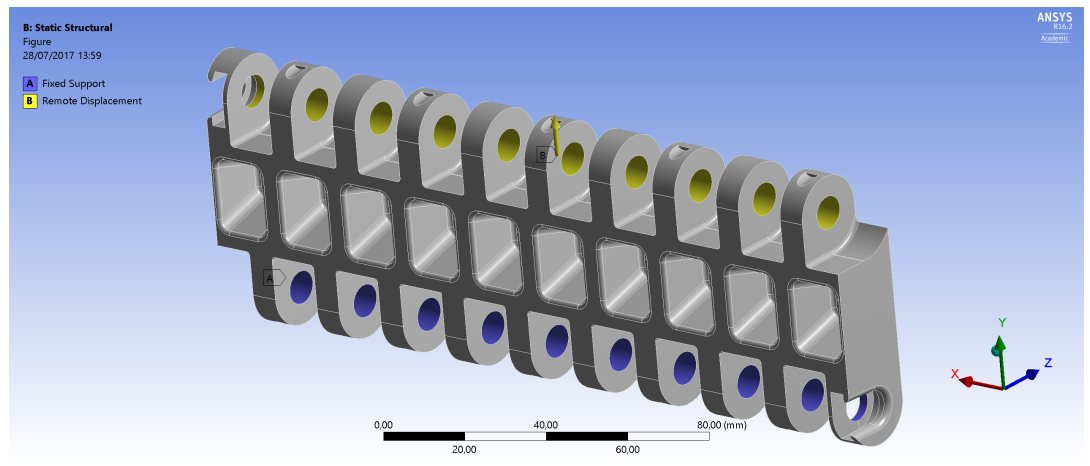

Figure 3: Applied load state

As the viscosimeter used works by capillary action, shear stress values $\tau$ and $\dot{\gamma}$ cannot be directly obtained. This is because as the melted material passes from a larger diameter to a smaller one, the pressure rises modifying the values slightly. The rheometer provided apparent values for viscosity and shear stress which were calculated using equations 1 and 2 , where $\tau_{a p}$ represents the apparent shear stress $(\mathrm{Pa}), \dot{\gamma}_{a p}$ is the apparent shear rate $\left(\mathrm{s}^{-1}\right), p$ is the pressure measured $(\mathrm{Pa})$, and $D$ and $L$ are the diameter $(\mathrm{mm})$ and the length $(\mathrm{mm})$ of the nozzle respectively.

$$
\begin{gathered}
\eta_{a p}=\frac{\tau_{a p}}{\dot{\gamma}_{a p}} \\
\tau_{a p}=\frac{p}{4 \cdot(L / D)}
\end{gathered}
$$

\section{${ }_{95}$ 2.5.1. Bagley's correction}

The flow of a material from a greater to a lesser diameter causes overpressure which slightly alters the results obtained from the capillary rheometer. This variation can be adjusted using Bagley's correction [8, which is shown in equation 3 , where $(L / D)_{c}$ is the $L / D$ ratio at zero pressure.

$$
\tau=\frac{p}{4\left((L / D)+(L / D)_{c}\right)}
$$

The application of Bagley's correction requires that a range of tests be carried out using different nozzles and shear rates in order to obtain a fit line between 
the pressure and the $L / D$ ratio, whose intersection with the $L / D$ axis gives us the $(L / D)_{c}$ value necessary for the correction. With this in mind, three different nozzles were used with a diameter of $1 \mathrm{~mm}$ and with L/D ratios of 10,20 and 30 , at shear rates of 100, 200, 500, 1000, 2000 and $5000\left(\mathrm{~s}^{-1}\right)$ at temperatures of 190 and $210{ }^{\circ} \mathrm{C}$.

\subsubsection{Rabinowitsch's correction}

Melted polymers are non-Newtonian fluids and thus the equations used for Newtonian fluids to calculate viscosity must also be adjusted. One of the most commonly used corrections is the Rabinowitsch [9] one, which makes it possible to calculate the real shear rate in the capillary wall using the Equation 4 , where $d \log \dot{\gamma}_{a p} / d \log \tau$ is the slope of the curve that relates the natural logarithm of $\dot{\gamma}_{a p}$ with $\tau$, calculated according to Equation 3

$$
\dot{\gamma}=\frac{\dot{\gamma}_{a p}}{4}\left(3+\frac{d \log \dot{\gamma}_{a p}}{d \log \tau}\right)
$$

\subsubsection{Determination of the material viscosity}

Once the real shear stress and shear rates were calculated, the viscosity values were obtained using equation 5 . This was done for each temperature.

$$
\eta=\frac{\tau}{\dot{\gamma}}
$$

With the aim of carrying out a later simulation of the injection process, the Cross model [10, which correlates the viscosity with the temperature, was used through equation 6 where $\eta_{0}$ (Pas) is the material viscosity under zero-shear-

rate conditions, $\tau^{*}(\mathrm{~Pa})$ represents the shear rate after which the pseudo-plastic behavior begins and $1-n$ represents the slope of the section with pseudo-plastic behavior. This slope can be calculated using the corrected measurements from the rheometer.

$$
\eta=\frac{\eta_{0}}{1+\left(\frac{\eta_{0}}{\tau^{*}} \cdot \dot{\gamma}\right)^{1-n}}
$$


The value of $\eta_{0}$ depends on the temperature, and it is therefore necessary to obtain this correlation before introducing this value in the Cross model. In order to do this, the Williams-Landel-Ferry 11 method was used, as can be seen in Equation 7, where $D_{1}$ (Pas) is the material viscosity at a null shear rate, at the vitreous transition temperature and an atmospheric pressure. $T$ is the temperature at which viscosity was planned to be attained, $T_{g}=223.15$ $\mathrm{K}$ is the vitreous transition temperature of the material, which was obtained using a power-compensation Differential Scanning Calorimeter (DSC) supplied by Mettler Toledo, S.A.E., model 821e, according to ISOs 11357-1 and 11357-4. $A_{1}(-)$ is a constant that depends on the material and $A_{2}=51.6 \mathrm{~K}$ is a constant which usually has a fixed value for all polymers [11.

$$
\eta_{0}=D_{1} \cdot e^{\frac{-A_{1}\left(T-T_{g}\right)}{A_{2}+\left(T-T_{g}\right)}}
$$
eters in the iteration $i, \eta_{j}$ and $\dot{\gamma}_{j}$ are the viscosity and shear speed of each of the points calculated previously from the readings from the rheometer, and $T$ represents each of the test temperatures. Finally, $\eta_{0_{i}}$ is the viscosity at a zero shear speed where the $i$ iteration is calculated from the dependent parameters.

The error minimization process is carried out using the fmincon function of Matlab ${ }^{\circledR}$ R2016, which allows restricted multi-variable minimizations to be solved, as the dependent variables cannot take any value. The "interior-point" algorithm was selected from different optimization algorithms available. 


\subsection{The mold injection analysis}

jection process was simulated. The injection mold was created in Solidworks ${ }^{\circledR}$ and provided by the manufacturer. The mold was then imported into Autodesk MoldFlow Insight ${ }^{\circledR} 2016$ software. Figure 4 shows the injection points used during simulation. The viscosity model was Cross-WLF, and the rheological properties were taken from test data (Table 3). The injection time (10 s), compaction time $(30 \mathrm{~s})$, and cooling temperature $\left(60{ }^{\circ} \mathrm{C}\right)$ were the same as used during the belt link manufacturing process.

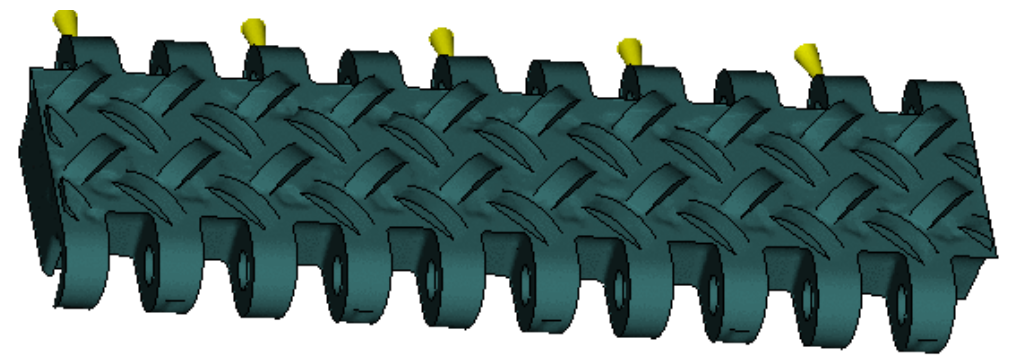

Figure 4: Mold injection model

\section{Results and discussion}

In order to determine the theoretical resistance of the belt link, a Finite Element Analysis was done. This established the maximum fracture load. Finally, an injection process simulation was carried out in order to determine whether the failure was due to the injection process used or the belt link design.

\subsection{Material tensile analysis}

After the injection of the specimens using the virgin POM provided by the belt link manufacturer, a series of five specimens were tested. Figure 5 and Table 1 show the results obtained with this analysis. As can be seen, the results are slightly different from those provided by the POM manufacturer. 


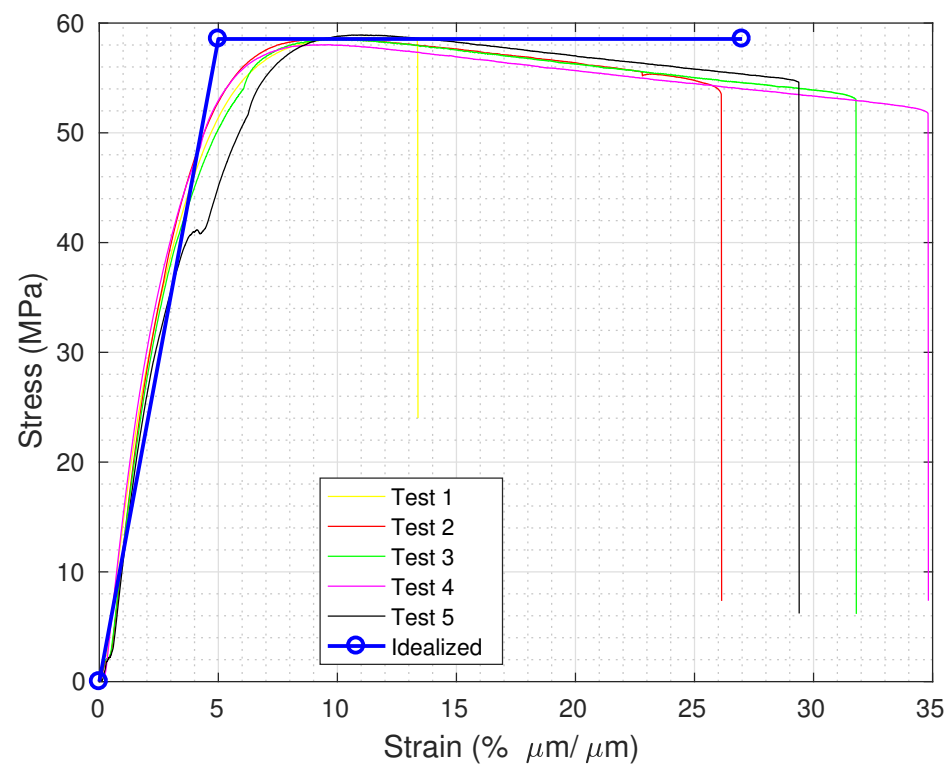

Figure 5: Tensile test of YUNKON ${ }^{\circledR}$ M90

Table 1: Tensile test data

\begin{tabular}{lccccc}
\hline Data & $\begin{array}{c}\bar{S}_{y}{ }^{1} \\
(\mathrm{MPa})\end{array}$ & $\begin{array}{c}S_{y_{\hat{\sigma}}{ }^{2}} \\
(\mathrm{MPa})\end{array}$ & $\begin{array}{c}\epsilon_{y}{ }^{3} \\
(\%)\end{array}$ & $\begin{array}{c}\bar{\epsilon}^{4} \\
(\%)\end{array}$ & $\begin{array}{c}\epsilon_{\hat{\sigma}}{ }^{5} \\
(\%)\end{array}$ \\
\hline Manufacturer & 61 & - & 9.4 & 35 & - \\
Tests & 58.55 & 0.33 & 9.0 & 27.12 & 8.31 \\
\hline
\end{tabular}

1 Yield strength. Average.

2 Yield strength. Standard deviation.

3 Yield strain.

${ }^{4}$ Break strain. Average.

${ }^{5}$ Break strain. Standard deviation.

\subsection{Belt link tensile test}

The tensile specimens results were obtained from ten tests, and one of them was discarded because of the pin premature breakage due to a inner defect. While the other specimens were being tested, the pins did not break prior to the belt links. Figure 6 and Table 2 show the results obtained in the tensile 
tests on the modular belt links. As can be seen, the values obtained are fairly dispersed for tensile strength and the associated displacement. The data also showed that the break began to occur under considerably lighter loads, reaching values as low as $700 \mathrm{~N}$. Finally, the majority of the breaks appeared in the lower part of the pin holes, as previously observed during in-service breakage.

These results show, without any doubt, that there are deficiencies associated with the design of the part, the material used, the manufacturing process, or even a combination of all these factors. Subsequently, a series of tests was developed to establish the source of the problem.

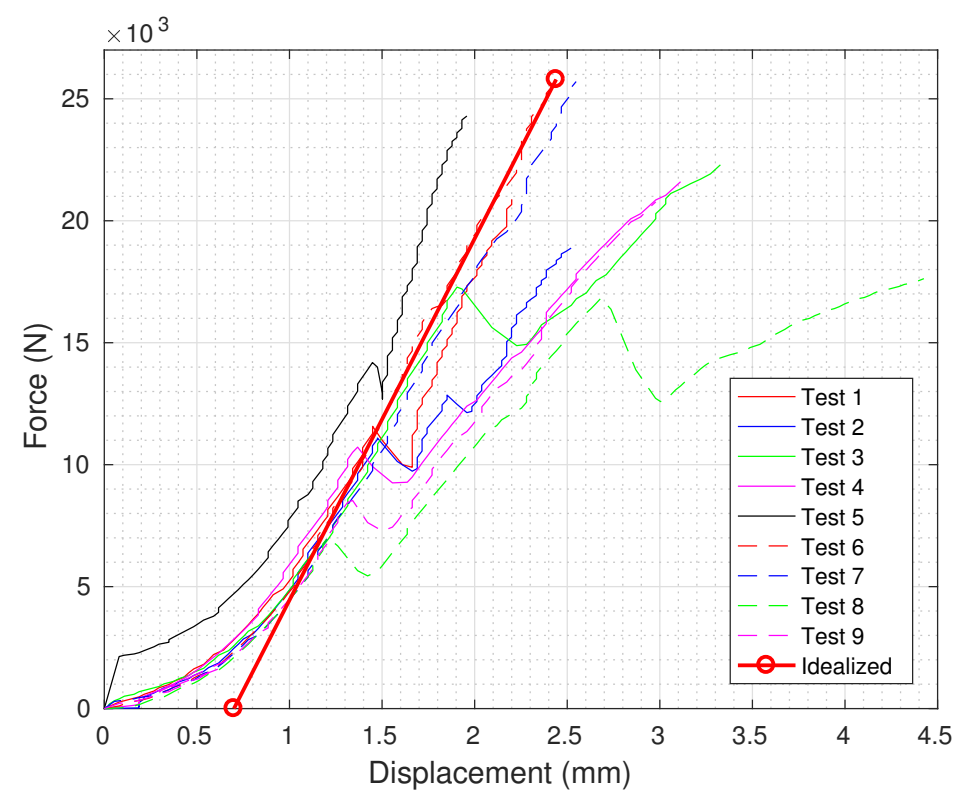

Figure 6: Belt link tensile tests results

Table 2: Tensile tests results

\begin{tabular}{lcccc}
\hline & Mean & Std. dev. & Max & Min \\
\hline Force $(\mathrm{N})$ & 21,982 & 2,855 & 25,787 & 17,627 \\
Displacement $(\mathrm{mm})$ & 2.835 & 0.74 & 4.426 & 1.958 \\
\hline
\end{tabular}




\subsection{Visual Inspection}

After carrying out the tensile tests on the modular belt, the breakages were analyzed. A simple visual inspection showed the divergence of the fracture shape (Figure 7), as well as the presence of internal imperfections (Figure 8). Figure 9 shows a more detailed analysis obtained under magnification. In all cases, there were imperfections in the fracture surface.

These types of imperfections have been reported previously by other authors 226], generally related to the manufacturing process and the study of stress concentration points that cause a later fracture [7, 12, 13].

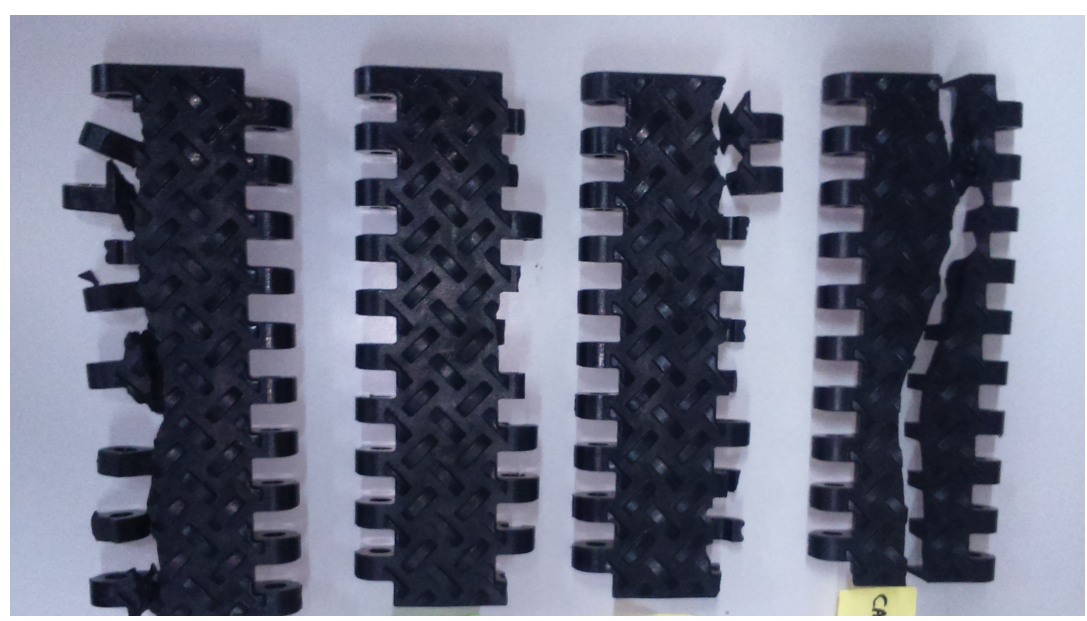

Figure 7: Comparative of different types of fracture.

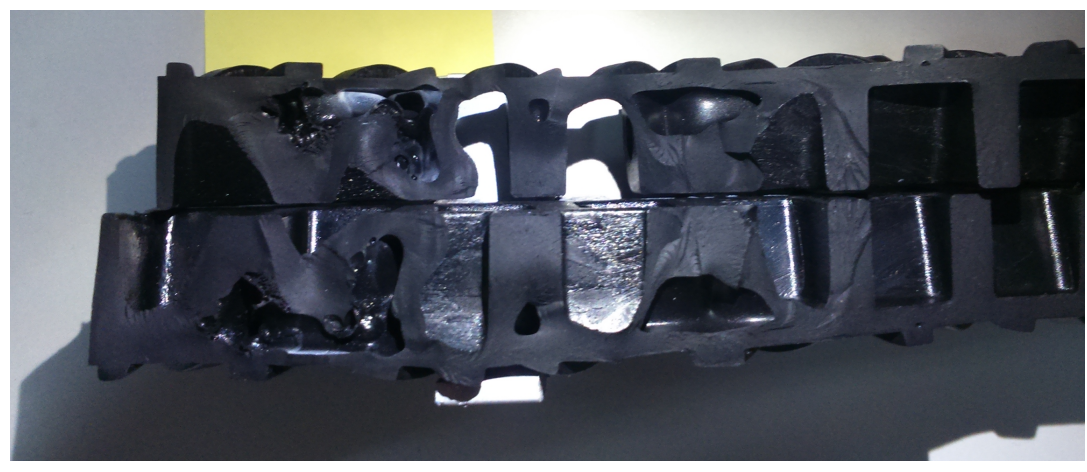

Figure 8: Part with internal imperfections. 

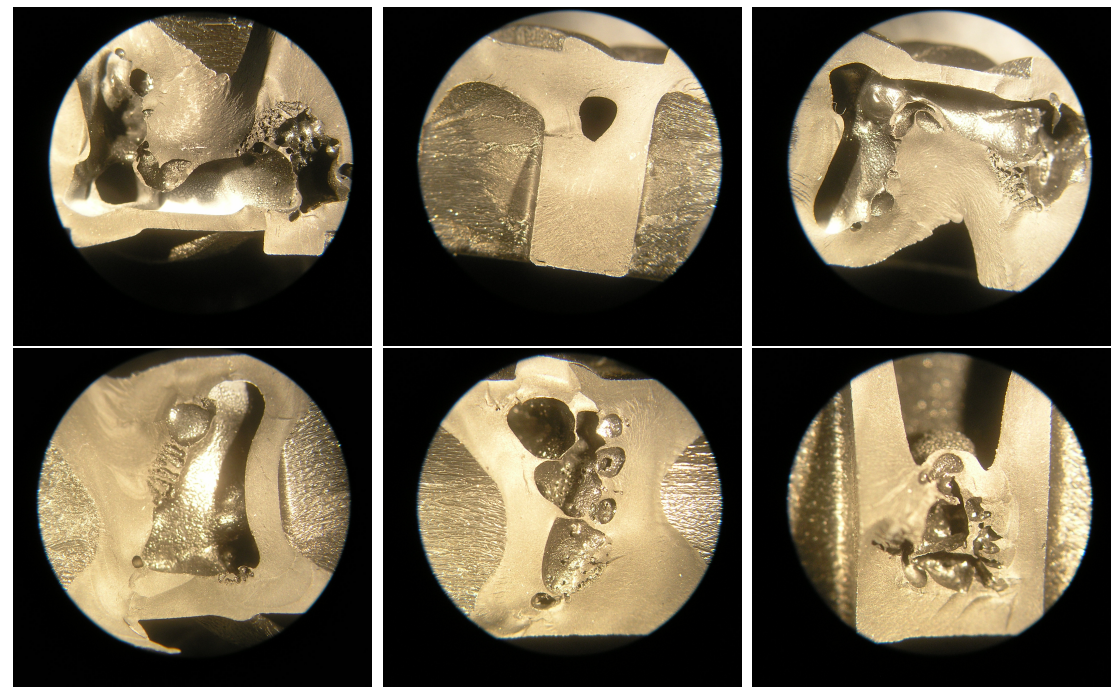

Figure 9: Detail of the internal imperfections at the frature zones.

\subsection{The Finite Element Analysis}

Finite element analysis (FEA) was performed on the belt link until the belt link elongation generated a strain beyond the strain at break measured in material tensile analysis. From the material tensile analysis (Table 1), the elastic deformation zone was idealized with a linear isotropic behavior with a Young modulus of 1,178 MPa and a Poisson ratio of 0.4 , while the plastic zone was modeled with isotropic plasticity with a yield stress of $58.55 \mathrm{MPa}$ and null tangent modulus (ideal plastic). Regarding to the load state, the lower holes were considered as fixed supports, while a remote displacement up to $4.5 \mathrm{~mm}$ (maximum displacement obtained in belt link tests, Table 2) was applied (Figure 3 .

Figures 10 and 11 show the stress and strain distribution at fracture initiation $(\sigma=58.55 \mathrm{MPa}$ and $\epsilon=0.271)$. The final fracture of the part is difficult to predict as, from the crack initiation the fracture mechanism follows a different behavior from the one predicted by the conventional elasticity theory. Thermoplastic polymers have a high strain at break point, so it is necessary to apply the Elastoplastic Fracture Mechanics theory (EPFM). This theory requires a more 
complex mathematical model, as well as more accurate test methods, such as the Essential Work of Fracture (EWF) method, in order to obtain parameters for the ductile fracture toughness process 14. The complexity of this analysis does not justify its use in this current study, so it was assumed that the theoretical fracture begins at the break strain, knowing that the theoretical tensile strength will be a little lower.

As can be seen in Figures 10 and 11, the most stressed and deformed region corresponds to the area where the tested belt links were broken, and where the majority of the internal imperfections were found. It is evident that the imperfections generated during the manufacturing process had a significant influence on the tensile strength of the part.

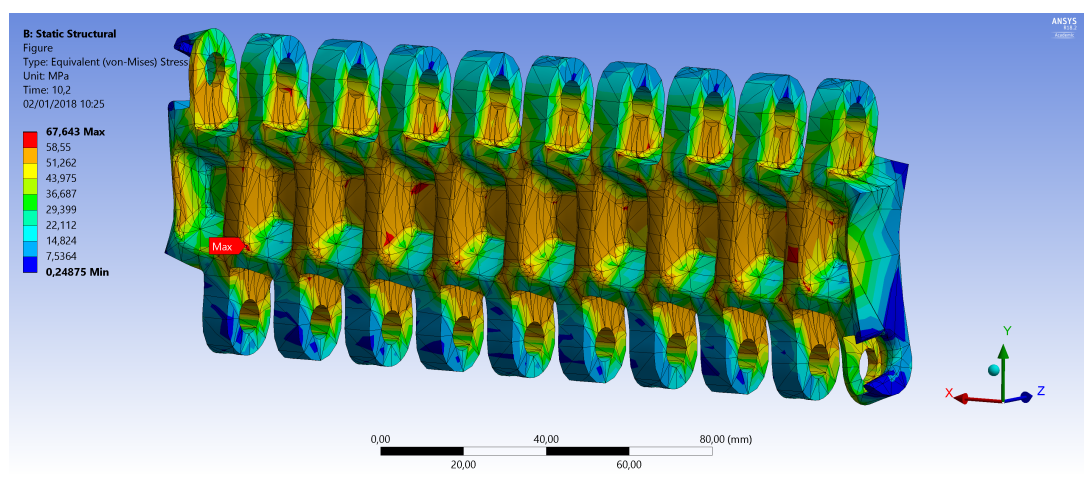

Figure 10: Von Mises stress distribution

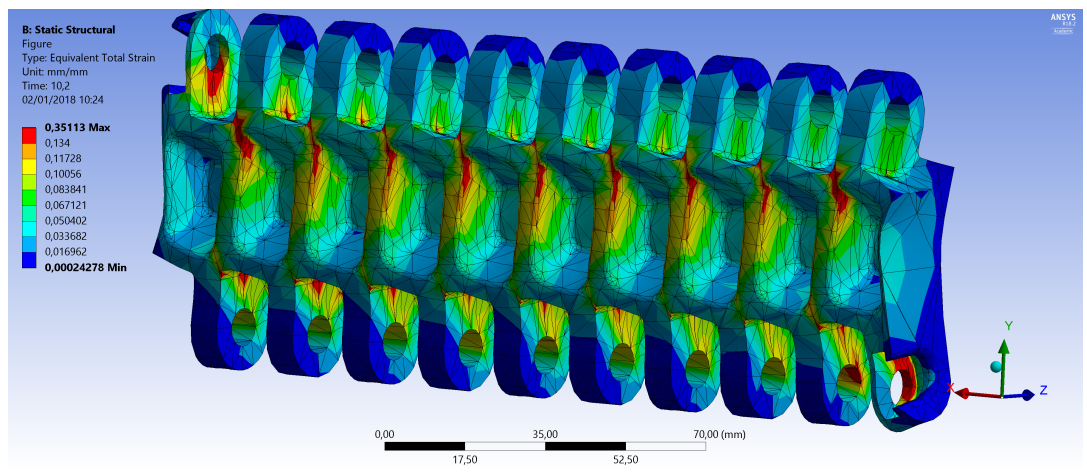

Figure 11: Strain distribution 
In order to evaluate strength loss associated with these internal defects, the real force and elongation on the belt link tests were compared with the results obtained from the simulation.

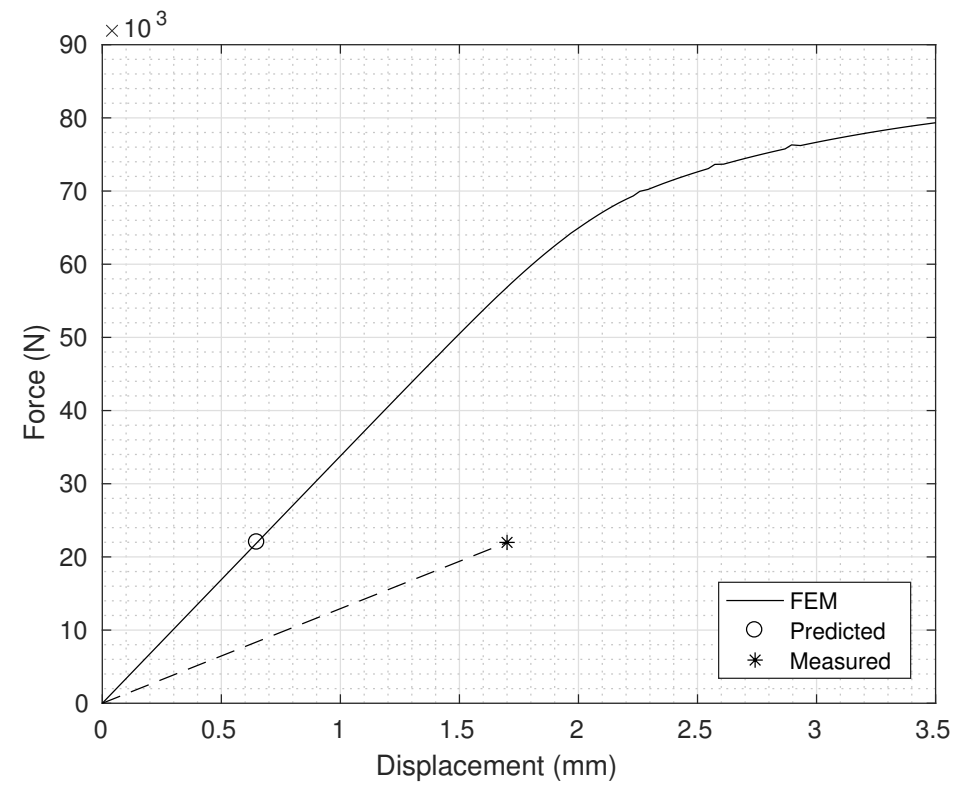

Figure 12: Force vs displacement

Figure 12 shows the relationship between force and displacement obtained from the FEA, as well as from the belt link tests (Figure6). As can be seen, the loss of stiffness is significant, reduced up to half the theoretical value. As the belt link actually works mainly under tensile efforts, this loss of stiffness means a useful section reduction of around 50\%, according to the results obtained using Equation 9 .

$$
K=\frac{F}{x}=\frac{A \cdot E}{L}
$$

As can be seen in Figure 13, the force required to reach the fracture strain, according to the specimen tests (from $13.4 \%$ to $34.8 \%$ ), oscillates between 58 and $78 \mathrm{kN}$. In terms of percentage, the dispersion of the fracture strain means a reduction of around $25 \%$ in the tensile strength of the belt link. 


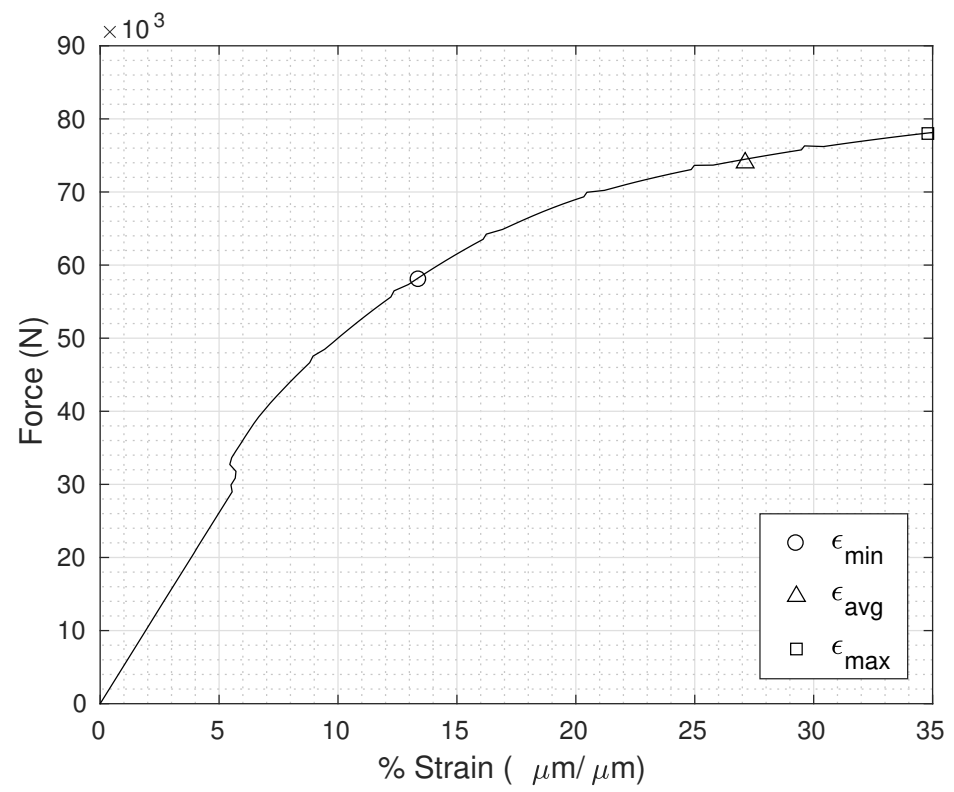

Figure 13: Force vs strain

On the other hand, if the theoretical average fracture force of the belt link $(74 \mathrm{kN})$ obtained for the average strain of the material $(27.12 \%)$ is compared with the average fracture force obtained in the belt link tests $(21,982 \mathrm{~N})$, there is a reduction of $70 \%$ in the belt link strength. This shows that, in addition to the dispersion introduced by the material itself, there must also exist an influence associated with the internal imperfections generated during the manufacturing process.

\subsection{Rheological characterization}

Table 3 and Figure 14 show the parameters and the behavior curve of the developed Cross-WLF model. Table 4 shows a summary of the rheological parameters from other POM manufacturers with a similar MFR. As can be seen, the obtained parameters are similar to those reported. 
Table 3: Cross-WLF model parameters for the YUNCON ${ }^{\circledR}$ M90 material

\begin{tabular}{ccclllc}
\hline$T$ & $n$ & $\tau^{*}$ & $D_{1}$ & $A_{1}$ & $\eta_{0}$ & $\begin{array}{c}\text { MFR } \\
(\mathrm{Pas})\end{array}$ \\
$(\mathrm{gr} / 10 \mathrm{~min})$
\end{tabular}

Table 4: Cross-WLF model parameters of other manufacturers. These values have been obtained from the Autodesk MoldFlow ${ }^{\circledR}$ database

\begin{tabular}{lcclcc}
\hline Reference & $\begin{array}{c}\tau^{*} \\
(-)\end{array}$ & \multicolumn{1}{c}{$\begin{array}{l}D_{1} \\
(\mathrm{~Pa})\end{array}$} & $\begin{array}{c}A_{1} \\
(\mathrm{Pas})\end{array}$ & $\begin{array}{c}\text { MFR } \\
(-)\end{array}$ \\
\hline Ultraform $\left.^{\circledR} \mathrm{gr} / 10 \mathrm{~min}\right)$ \\
Hostaform $^{\circledR}$ C9021 M & 0.2741 & 305,000 & $9.62 \cdot 10^{13}$ & 30.367 & 8.5 \\
Celcon $^{\circledR}$ MC90 & 0.1507 & 326,633 & $7.34 \cdot 10^{14}$ & 32.809 & 8.7 \\
Kepital $^{\circledR}$ F20-03 & 0.3475 & 68,600 & $9.96 \cdot 10^{11}$ & 24.306 & 9.0 \\
Tanoform $^{\circledR} 300$ & 0.1982 & 293,348 & $3.65 \cdot 10^{12}$ & 27.186 & 9.0 \\
\hline
\end{tabular}

\subsection{The mold injection analysis}

Once the reduction in strength associated with the fracture was quantified, the next step was to determine whether the part failure was due in part to an error in design. With this objective in mind, and using the rheological test data, the injection process was simulated using the Autodesk MoldFlow Insight ${ }^{\circledR} 2016$ software.

During the development of the injection process model, a replica of the original mold was made, using five injection points, as well as the original cooling system. This can be seen in Figure 15. The next step was to simulate the mold filling to create the part. The filling was started at all five points simultaneously. As can be seen in Figure 15, the use of multiple injection points produced interactions between the material, which created residual tension, as well as 


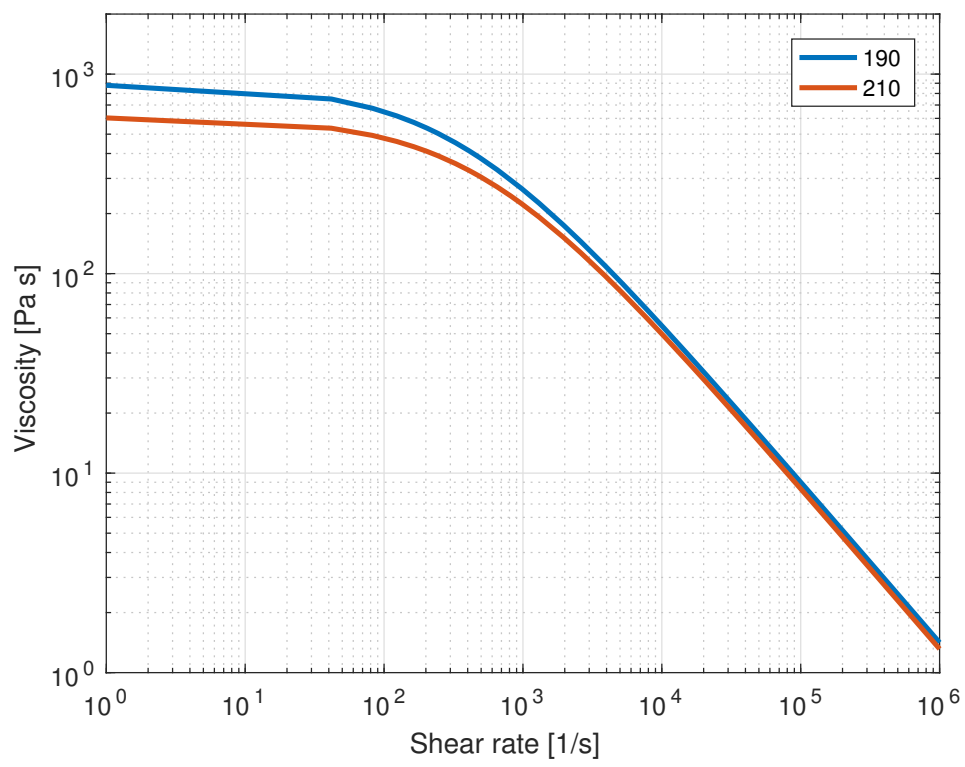

Figure 14: $\mathrm{YUNCON}^{\circledR}$ viscosity at $190{ }^{\circ} \mathrm{C}$ and $210^{\circ} \mathrm{C}$

joint lines.

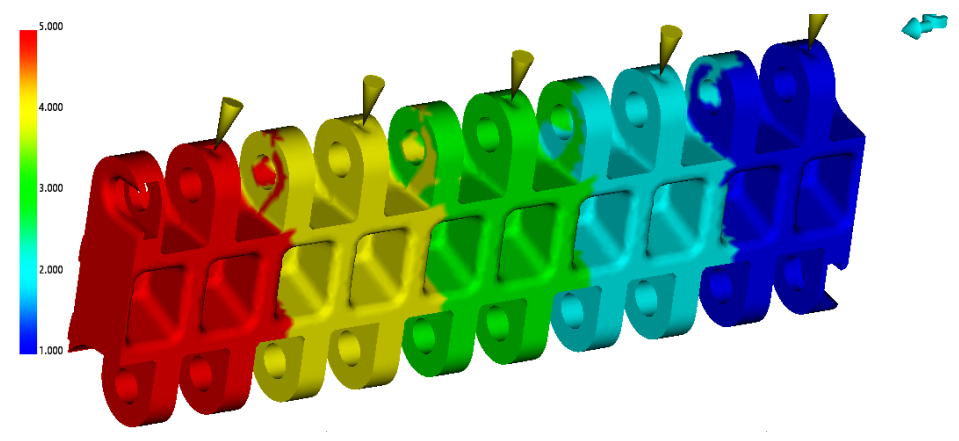

Figure 15: The mold filling efficiency

Figure 16 shows the shrinkage of the belt link, which is quite high, reaching up to $2.5 \mathrm{~mm}$. This shrinkage is basically due to the high thickness of the part as well as the high shrinkage percentage of the material $(2.1 \%)$.

Looking at Figure 17 and Figure 18, it is possible to see that the zones with maximum suckbacks correspond to the weld lines, so the material becomes 

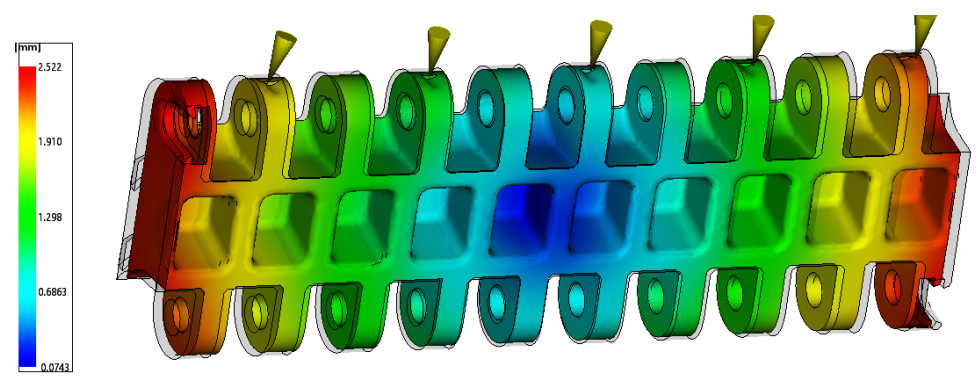

Figure 16: Part shrinkage

weaker in these areas [15 17. On the other hand, the high thickness of the part causes a high temperature gradient of the material during the cooling, so when the outer part of the material solidifies, while the inner part is still melted. As the inner part cools, it also shrinks, and if there is no new provision of material because the outer part has solidified, the volumetric shrinkage of the inner part leads to inner holes known as suckbacks. Finally, the changes in the flow direction as the mold is filled, can cause bad orientation of the polymeric chains, and may even result in some chains being arranged in a perpendicular direction to the load action. This would also significantly reduce the material strength.

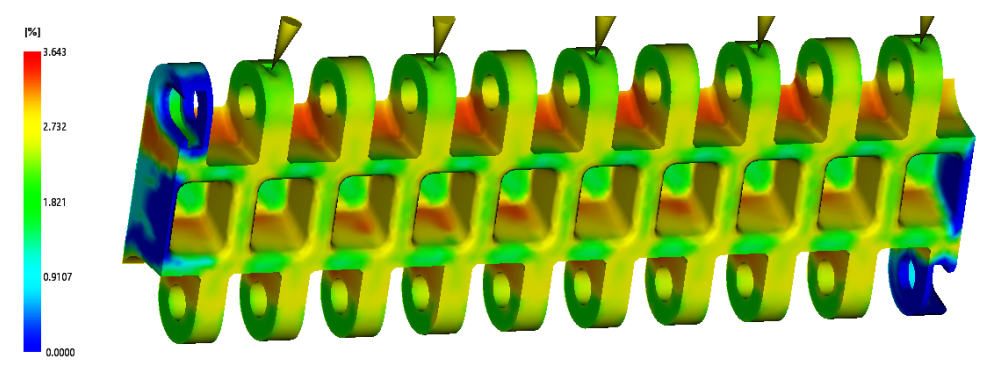

Figure 17: Internal suckbacks

Finally, Figure 19 shows the area where the simulation predicts the formation of air traps, which are produced by the thick/thin/thick change in thickness causing changes in the flow rate due to venturi effect. On the other hand, a large degree of thickness prevents the correct elimination of the air traps. 


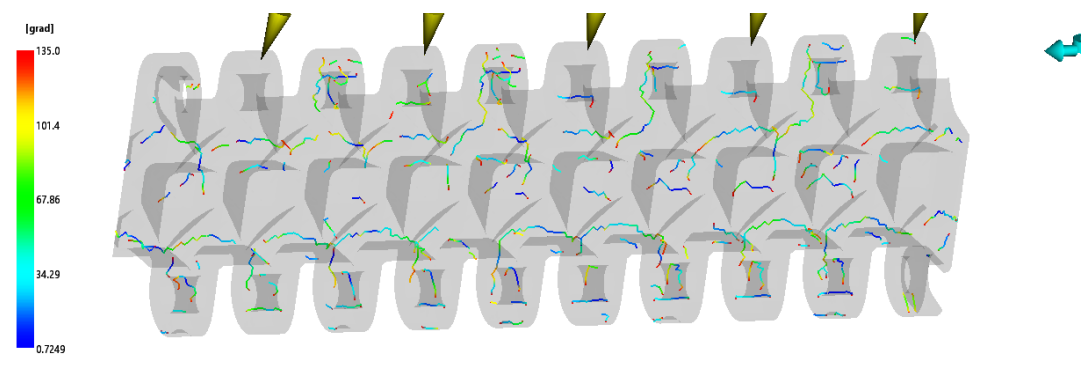

Figure 18: Weld lines

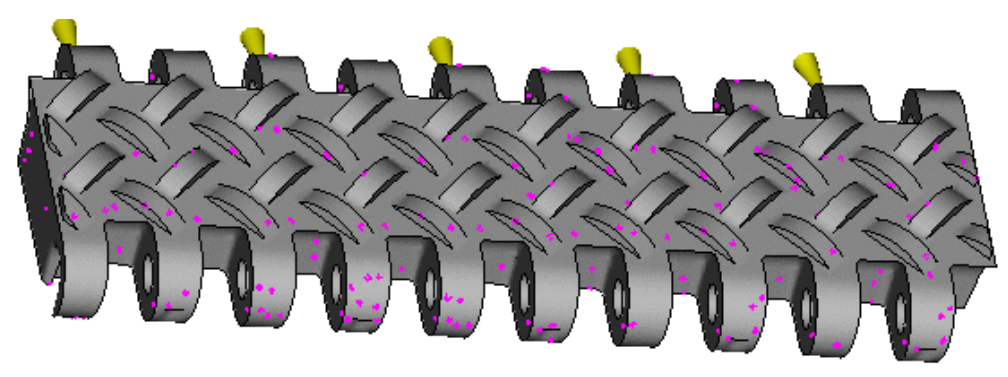

Figure 19: Air traps

As can be seen in Figure 19, the internal defects detected in the broken tested belt links are located in the zone where the simulation predicted trapped air bubbles, gaps, internal suckbacks and weld lines. These same zones are the most stressed ones according to the earlier FEM analysis, and thus the combination of both factors produces a significant loss of strength. It can therefore be deduced that the failure of the belt link was due not to an error in the processing of the material, but rather in the design of the part.

\section{Conclusions}

Based on the results of this study it has been possible to determine that the failure of the modular plastic belt was not due to an overload, to the material, or to the manufacturing process, but was in fact due to the poor design of the part. This conclusion is taken from the mechanical characterization of the material, as well as the strain simulation carried out using FEA and the analysis of the manufacturing process. The stiffness reduction in the tested belt links reaches 

compared with the theoretical values. This variation can not be attributed solely to the variability in the mechanical characteristics as it only has an influence of $25 \%$ on the theoretical tensile strength.

The simulation of the injection process showed how a set of unfavorable factors caused the inner defects in the parts. These factors cannot be attributed to the material supplied by the manufacturer, as it has a rheology similar to other manufacturers. Neither can the defects be attributed to material type. The conclusive result of this study is that the failure of the belt links was due to the geometry of the part itself, where significant shrinkage, weld-lines, the zig-zagging of the flow and the thickness variation of the melted material led to the failure of the part. Regarding possible solutions to the problem, changing the material is not a valid option. Although the use of other materials with a greater Melt Flow Rate such as polypropylene could reduce some of these adverse effects, they also bring their own set of disadvantages, such as a reduction in life of the part.

Thus, the only viable option to avoid the formation of these defects is to redesign the belt link and the mold, following the directions stated below:

- Reduce the thicknesses and make them uniform. With a reduction in thick-

- Addition of fillers into the material. Although the addition of fillers would reduce the Melt Flow Rate, it would decrease the shrinkage associated with cooling after the injection process. This, in turn, would yield a reduction in internal air traps, and would prevent the appearance of suckbacks. 


\section{Acknowledgements}

The authors would like to acknowledge the support of Mr. Luke Napierkowski.

[2] A. Vaxman, M. Narkis, A. Siegmann, S. Kenig, Void formation in shortfiber 325

[6] F. Zakar, M. Budinski, Fracture of a saddle fusion (weld) joint in high density polyethylene (hdpe) pipe, Engineering Failure Analysis 82 (2017) 481 - 492. doi:https://doi.org/10.1016/j.engfailanal.2017.03.009

[7] M. Horstemeyer, J. Lathrop, A. Gokhale, M. Dighe, Modeling stress state dependent damage evolution in a cast al-si-mg aluminum alloy, Theoretical and Applied Fracture Mechanics 33 (1) (2000) 31-47. 
[8] E. Bagley, End corrections in the capillary flow of polyethylene, Journal of Applied Physics 28 (5) (1957) 624-627. doi:10.1063/1.1722814.

[9] B. Rabinowitsch, Uber die viskositat und elastizitat von solen, Zeitschrift fur Anorganische und Allgemeine Chemier Physikalische Chemie 145A.

[10] M. Cross, Rheology of non-newtonian fluids: A new flow equation for pseudoplastic systems, Journal of Colloid Science 20 (5) (1965) 417-437. doi : 10.1016/0095-8522(65)90022-X.

[11] M. Williams, R. Landel, J. Ferry, The temperature dependence of relaxation mechanisms in amorphous polymers and other glass-forming liquids, Journal of the American Chemical Society 77 (14) (1955) 3701-3707. doi:10.1021/ja01619a008.

[12] A. Cocks, M. Ashby, On creep fracture by void growth, Progress in Materials Science 27 (3-4) (1982) 189-244. doi:10.1016/0079-6425(82) 90001-9

[13] M. Brunig, S. Gerke, V. Hagenbrock, Stress-state-dependence of damage strain rate tensors caused by growth and coalescence of micro-defects, In-

1 ternational Journal of Plasticity 63 (2014) 49-63. doi:https://doi.org/ $10.1016 / j$.ijplas.2014.04.007.

[14] A. Martinez, J. Gamez-Perez, M. Sanchez-Soto, J. Velasco, O. Santana, M. L. Maspoch, The essential work of fracture (ewf) method - analyzing the post-yielding fracture mechanics of polymers, Engineering Failure Analysis 16 (8) (2009) 2604 - 2617. doi:10.1016/j.engfailanal.2009.04.027.

[15] C.-H. Wu, W.-J. Liang, Effects of geometry and injection-molding parameters on weld-line strength, Polymer Engineering and Science 45 (7) (2005) 1021-1030. doi:10.1002/pen.20369.

[16] R. Selden, Effect of processing on weld line strength in five thermoplastics, Polymer Engineering and Science 37 (1) (1997) 205-218. 
[17] S. Fellahi, A. Meddad, B. Fisa, B. Favis, Weldlines in injection molded parts: A review, Advances in Polymer Technology 14 (3) (1995) 169-195. doi:10.1002/adv.1995.060140302 